J. Klin. Endokrinol. Stoffw. 2021 · 14:2-10 https://doi.org/10.1007/s41969-021-00131-2 (c) Springer-Verlag GmbH Austria, ein Teil von Springer Nature 2021

\title{
Jahrestagung der Österreichischen Gesellschaft für Endokrinologie und Stoffwechsel (ÖGES) gemeinsam mit der Österreichischen Schilddrüsengesellschaft (OSDG) und der Austrian Neuroendocrine Tumor Society (ANETS)
}

\author{
22.-24. April 2021, virtuell
}

\section{ÖGES Abstracts (in alphabetischer Reihenfolge der präsentierenden Autoren)}

\section{Neurocognitive functions in chronic hypoparathyroidism Scherkl M. ', Tmava-Berisha A. ', Fahrleitner-Pammer A. ', Amrein K. ${ }^{* 1}$ 'Medizinische Universtität Graz, Graz, Österreich}

Background: Chronic hypoparathyroidism is a rare endocrine disorder characterized by inadequately low parathyroid hormone (PTH) levels and hypocalcemia. Possible late manifestations of the consecutive calcium and phosphate disturbance are severe and may lead to intracranial calcifications and neurocognitive impairment. Affected patients report frequent brain fog, depressive symptoms, cognitive impairment and a reduced quality of life.

Methods: In this thesis, a battery of neurocognitive tests including the Mini-Mental-State-Examination (MMSE), the Multiple-Choice-Vocabulary-Test (MWT), and the Munich-Memory-Test (MMT) was used to assess general cognitive functions. In the period from 2019 to 2021, 10 adult patients with diagnosed hypoparathyroidism could be identified as study participants and underwent neurocognitive assessment.

Results: 9/10 study participants are women. The mean age at onset of the disease was $33.3( \pm 9.9)$ years, and the neurocognitive assessment was performed after an average of $8.5( \pm 8.6)$ years of disease duration.

The global MMSE score of the study group of $29.5( \pm 0.7)$ was similar to the control group with $29.7( \pm 0.6)$ points. None of the study participants had a score below the threshold of 24, which would indicate impaired cognitive function. According to the MWT, the mean estimated IQ score of the study group was $108.4( \pm 9.9)$ and therefore above the median raw score of the norming sample, defined as 100 . The statistical evaluation of the test results showed no correlation between chronic hypoparathyroidism and neurocognitive dysfunction.

Conclusion: There is currently only limited data available on the pathophysiological link between chronic hypoparathyroidism and neurocognitive dysfunction. Further studies including functional MRI testing need to provide laboratory and neuroimaging evidence along with neurocognitive assessment to support an association between PTH deficiency and impaired cognitive functions. Also, the effects of PTH replacement should be addressed.

\section{Project „Backtoclinic I“: An overview on the state of care of adult PKU patients in Austria}

Beghini M. ${ }^{* 1}$, Resch F. J. ${ }^{1}$, Möslinger D. ${ }^{2}$, Konstantopoulou V. ${ }^{2}$, Karall D. ${ }^{3}$, Brunner-Krainz M. ${ }^{4}$, Plecko B. ${ }^{4}$, Spenger J. ${ }^{5}$, Kautzky-Willer A. ${ }^{1}$, Scherer T. ${ }^{1}$, Hufgard-Leitner M.

'Division of Endocrinology and Metabolism, Department of Internal Medicine III, Medical University of Vienna, Vienna, Austria, ${ }^{2}$ Department of Paediatrics and Adolescent Medicine, Medical University of Vienna, Vienna, Austria, ${ }^{3}$ Division of Inherited Metabolic Disorders, Clinic of Pediatrics I, Medical University of Innsbruck, Innsbruck, Austria, ${ }^{4}$ Division of General Pediatrics, Department of Pediatrics and Adolescent Medicine, Medical University of Graz, Graz, Austria, ${ }^{5}$ University Children's Hospital, Salzburger Landeskliniken (SALK) and Paracelsus Medical University (PMU), Salzburg, Austria

Background: High rates of lost to follow-up (LTFU) adult patients are a major concern in the long-term management of phenylketonuria (PKU). To address this issue, we designed the project "Backtoclinic I" with the purpose of identifying LTFU adult PKU patients in Austria as a first step to reestablish appropriate treatment.

Subjects and methods: Individuals born between 1966 and 1999 and diagnosed with PKU through the National Austrian Newborn Screening Program (NANSP) were identified using the NANSP's database. Follow-up data were collected in the Austrian metabolic centers (Medical University of Vienna, Graz, Innsbruck and Salzburg). Patients with no contact to any of these centers within the last two years were classified as LTFU. Epidemiological characteristics of the whole study population as well as of LTFUand currently in follow-up patients were analyzed.

Results: between 1966 and 1999, 281 newborns were diagnosed with PKU through the NANSP. Two patients died in their first year of life and were excluded from the analysis. Of the remaining 279 patients (mean age \pm SD: $36.7 \pm 9.1$ y, $42.7 \%$ females), $177(63.4 \%)$ are LTFU. The rate of LTFU patients is higher in men than in women $(68.1 \%$ vs $57.5 \%)$, and markedly increases with age in both sexes. The gender gap is greatest in young adults ( $52.6 \%$ vs. $25.0 \%$ in the age range $20.0-24.9$ y) and declines with age $(94.4 \%$ vs. $80.0 \%$ in age $>45.0 \mathrm{y}$ ). 
Conclusions: We found an alarming rate of $63.4 \%$ of LTFU PKU adult patients in Austria, and observed, for the first time, a gender gap in the PKU state of care. Our findings illustrate the urgent need for the metabolic community to identify LTFU adult PKU patients and to develop strategies to reestablish appropriate treatment for men and women with PKU.

\section{Geschlechtsspezifische Mortalitäts-Unterschiede bei Patientinnen und Patienten nach bariatrischer Operation - Eine bevölkerungsbezogene Datenanalyse}

Beiglböck H. ${ }^{* 1}$, Mörth E. ${ }^{2}$, Reichardt B. ${ }^{3}$, Stamm T. ${ }^{4}$, Itariu B. ${ }^{1}$, Harreiter J.', Hufgard-Leitner M. ', Fellinger P.', Eichelter J., Prager G. ${ }^{5}$, Kautzky-Willer A. ', Wolf P.', Krebs M.

'Division of Endocrinology and Metabolism, Department of Internal Medicine III, Medical University of Vienna, Wien, Österreich, ${ }^{2}$ Mohn Medical Imaging and Visualization Centre, Haukeland University Hospital, Bergen, Norwegen, ${ }^{3}$ Österreichische Gesundheitskasse, Eisenstadt, Österreich, ${ }^{4}$ Center for Medical Statistics, Informatics and Intelligent Systems, Institute for Outcomes Research, Medical University of Vienna, Wien, Österreich, ${ }^{5}$ Division of General Surgery, Department of Bariatric Surgery, Medical University of Vienna, Wien, Österreich

Fragestellung: Die Bariatrische Chirurgie ist eine effiziente Therapiestrategie für die Behandlung morbider Adipositas. Ob verstorbene Patientinnen und Patienten mit einer bariatrischen Operation einen geschlechtsspezifischen Unterschied im Hinblick auf Komorbiditäten sowie Todesursachen aufweisen, ist nicht abschließend geklärt. Das Ziel dieser bevölkerungsbasierten Datenanalyse war es, diese Unterschiede zu beleuchten.

Material und Methoden: Die Versicherungsdaten der österreichischen Gesundheitskasse (ÖGK) von allen Patientinnen und Patienten mit einer bariatrischen Operation zwischen Jänner 2010 und Dezember 2018 wurden analysiert. Insgesamt konnten 19.901 Patientinnen und Patienten mit 107.806 Beobachtungsjahren eingeschlossen werden. Komorbiditäten von Verstorbenen wurden anhand von ICD-Codes und Medikamenteneinnahmen basierend auf ATC Codes klassifiziert. Folgende Adipositas assoziierten Komorbiditäten wurden analysiert: Diabetes mellitus (DM), kardiovaskuläre Erkrankungen (CV), psychiatrische Erkrankungen (PSY) und Krebserkrankungen $(\mathrm{K})$.

Ergebnis: Das durchschnittliche Alter zum Operationszeitpunkt in der gesamten Kohorte war 40,6 \pm 12,5 Jahre (Männer: 41,8 \pm 12,6, Frauen: $40,1 \pm 12,4 ; p<0,001)$. Nach einer mittleren Beobachtungszeit von 5,4 $\pm 2,6$ Jahren waren 367 (1,8\%) Patientinnen und Patienten innerhalb der Beobachtungszeit von Jänner 2010 bis April 2020 verstorben. Die Todesrate pro Beobachtungsjahr war 0,34\% und bei Männern signifikant höher als bei Frauen (Männer: 0,64 \%; Frauen: 0,24 \%). Die 30-Tage-Mortalität war $0.2 \%$ und bei Männern fünfmal höher verglichen zu Frauen $(0,1 \%$ vs. $0,5 \%, p<0,001)$. Unabhängig vom Geschlecht waren psychiatrische Erkrankungen (47\%) und kardiovaskuläre Erkrankungen (48\%) die häufigsten Komorbiditäten bei den Verstorbenen. Krebserkrankungen und Diabetes waren bei $33 \%$ und $36 \%$ vorhanden.

Schlussfolgerung: Diese Datenanalyse zeigt eine fünfmal höhere 30-Tage-Mortalität nach bariatrischer Operation bei Männern im Vergleich zu Frauen. Bei der Langzeitmortalität zeigte sich ebenfalls eine erhöhte Mortalität bei Männern. Krebserkrankungen und Infektionen waren die häufigsten Todesursachen.

\section{Patients with congenital adrenal hyperplasia show an adverse cardiovascular risk profile compared to patients with autoimmune adrenalitis}

Beiglböck H. ${ }^{* 1}$, Bögl M. ${ }^{1}$, Fellinger P. ${ }^{1}$, Metz M. ${ }^{\prime}$, Vila G. ', Luger A. ', Trattnig S. ${ }^{1}$, Kautzky-Willer A. ${ }^{\prime}$, Krssak M.', Krebs M. ${ }^{1}$, Wolf P. ${ }^{\prime}$

'Division of Endocrinology and Metabolism, Department of Internal Medicine III, Medical University of Vienna, Vienna, Austria

Background: Despite adequate hormone replacement therapy, evidence suggests an increased mortality in patients suffering from primary adrenal insufficiency, mainly because of cardiovascular diseases. Congenital adrenal hyperplasia $(\mathrm{CAH})$ and autoimmune adrenalitis $(\mathrm{AI})$ are two entities with a different pathophysiological background and might therefore show divergent cardiovascular risk profiles.

Methods: 9 patients with CAH (female $n=4$; age: $39 \pm 11$ years; weight: $71 \pm 12 \mathrm{~kg}$; equivalent dose of hydrocortisone: $19 \pm 7 \mathrm{mg} /$ day) and $9 \mathrm{pa}-$ tients with AI (female $n=4$; age: $43 \pm 9$ years; weight: $73 \pm 15 \mathrm{~kg}$; equivalent dose of hydrocortisone: $24 \pm 7 \mathrm{mg} /$ day) under stable glucocorticoid substitution therapy were investigated. Fasting blood was drawn to evaluate glucose- and lipid metabolism. Standardized blood pressure measurements and magnetic resonance imaging and spectroscopy measurements were performed to assess cardiac function, myocardial (MYCL) \& hepatic lipid content (HCL) and abdominal fat mass.

Results: Fasting glucose $(91 \pm 10$ vs $80 \pm 7 \mathrm{mg} / \mathrm{dl} ; \mathrm{p}=0.021), \mathrm{HbAlc}$ $(5.44 \pm 0.3$ vs $5.1 \pm 0.4 \% ; p=0.044)$ and blood pressure $(124 \pm 8 / 80 \pm 6$ vs $113 \pm 10 / 71 \pm 6 ; p=0.031$ ) were higher in CAH compared to AI. This was paralleled by an increase in HCL ( $5.8 \pm 5$ vs $1.8 \pm 1 \%$; $p=0.0549)$ and abdominal fat mass $(251 \pm 130$ vs $378 \pm 119 \mathrm{~mm} 2 ; p=0.047)$. No differences in heart function (Ejection fraction: $56 \pm 4$ vs $59 \pm 8 \%$; $p=$ n. s.) and MYCL $(0.28 \pm 0.2$ vs $0.25 \pm 0.1 \% ; p=$ n. s. $)$ were observed. Patients with CAH were treated more frequently by evening doses of glucocorticoids ( $6 / 9 \mathrm{vs} 1 / 9)$ or others than hydrocortisone (5/9 vs 0/9).

Conclusions: $\mathrm{CAH}$ is associated with an adverse cardiovascular risk profile compared to AI, despite a comparable dose of daily glucocorticoid substitution. This might be explained by a more unphysiological timing of replacement therapy or the more frequent use of longer-acting glucocorticoids in patients with $\mathrm{CAH}$, as well as by the adverse metabolic effects of hyperandrogenemia in women.

\section{Einfluss von (Früh-) Schwangerschaften nach bariatrischer Operation auf die Kindergesundheit - Eine bevölkerungsbezogene Datenanalyse}

Beiglböck H. ${ }^{* 1}$, Mörth E. ${ }^{2}$, Reichardt B. ${ }^{3}$, Stamm T. ${ }^{4}$, Itariu B. ${ }^{1}$, Harreiter J. ${ }^{1}$, Hufgard-Leitner M. ', Fellinger P.', Eichelter J. ${ }^{5}$, Prager G. ${ }^{5}$, Kautzky-Willer A. ${ }^{\prime}$ Wolf P. ${ }^{1}$, Krebs $M$.

'Division of Endocrinology and Metabolism, Department of Internal Medicine III, Medical University of Vienna, Wien, Österreich, ${ }^{2}$ Mohn Medical Imaging and Visualization Centre, Haukeland University Hospital, Bergen, Norwegen, ${ }^{3}$ Österreichische Gesundheitskasse (ÖGK), Eisenstadt, Österreich, ${ }^{4}$ Center for Medical Statistics, Informatics and Intelligent Systems, Institute for Outcomes Research, Medical University of Vienna, Wien, Österreich, ${ }^{5}$ Division of General Surgery, Department of Bariatric Surgery, Medical University of Vienna, Wien, Österreich

Fragestellung: Gewichtsmodifizierende Operationen haben einen nachgewiesenen Effekt auf die Fertilität von Frauen. Während der ersten Phase der Gewichtsabnahme ist eine Schwangerschaft allerdings nicht empfohlen, damit möglicherweise auftretende negative Einflüsse auf das Ungeborene reduziert werden können. Das Ziel dieser bevölkerungsbasierten Datenanalyse war es zu analysieren, ob der Zeitraum zwischen bariatrischer Operation und Entbindung einen Zusammenhang mit der Kindergesundheit zeigt.

Material und Methoden: Die Versicherungsdaten der österreichischen Gesundheitskasse (ÖGK) von allen Patientinnen mit einer bariatrischen Operation zwischen Jänner 2010 und Dezember 2018 wurden analysiert. Insgesamt konnten 14.688 Patientinnen eingeschlossen werden. Das Alter zum Zeitpunkt der Operation, die verstrichene Zeit von der Operation bis zur Geburt sowie die Krankenhaustage der Kinder wurden ausgewertet. Ergebnis: Insgesamt wurden 1057 Patientinnen (7\%) innerhalb des Beobachtungszeitraumes schwanger. Bei diesen wurden 1369 Geburten (männlich: 766 weiblich: 633) registriert. Im Mittel erfolgten die Geburten 39,4 $\pm 21,9$ Monaten postoperativ. Das Alter der Mütter zum Zeitpunkt der Operation war 27,1 $\pm 4,9$ Jahre. 70 Schwangerschaften (5\%) innerhalb von 12 Monaten nach der Operation wurden beobachtet. Innerhalb von 18 Monaten postoperativ konnten 252 (18\%) Geburten detektiert werden. Fast jede dritte Geburt (31 \%) erfolgte innerhalb von 24 Monaten nach der 
bariatrischen Operation. Bei der Anzahl der Krankenhaustage der Kinder zeigte sich kein signifikanter Unterschied zwischen Kinder, die vor/nach 12 Monaten postoperativ ( $1,8 \pm 4,6$ vs. $2,7 \pm 11,3$ Tage), vor/nach 18 Monaten postoperativ $(2,7 \pm 10,2$ vs. $2,6 \pm 11,3$ Tage) und vor/nach 24 Monaten postoperativ (2,2 $\pm 8,2$ vs. $2,8 \pm 12,1$ Tage) geboren wurden.

Schlussfolgerung: Die vorläufige Datenanalyse zeigt, dass Schwangerschaften innerhalb von 24 Monaten nach einer bariatrischen Operation häufig sind. Die Anzahl der Krankenhaustage der Kinder scheint dadurch jedoch nicht beeinflusst zu sein.

\section{Vergleich der Inzidenz von Nebennierenkrisen bei Patientinnen und Patienten mit primärer und sekundärer Nebenniereninsuffizienz}

Bögl M. S. ${ }^{* 1}$, Beiglböck H. ', Fellinger P. ', Krebs M. ', Luger A. ', Vila G. ', KautzkyWiller A. ', Looser C. ', Wolf P.'

'Medizinische Universität Wien, Klinik für Innere Medizin III, Abteilung für Endokrinologie und Stoffwechsel, Wien, Österreich

Fragestellung: Nebennierenkrisen sind potentiell lebensbedrohliche Notfälle für PatientInnen mit bestehender Glukokortikoider (GK) Substitutionstherapie aufgrund einer primären oder sekundären Nebenniereninsuffizienz (NNI). Durch eine mögliche adrenale Restfunktion sowie der unbeeinträchtigten Mineralokortikoid-Regulation könnte sich das Risiko für Nebennierenkrisen bei sekundärer NNI unterscheiden. Dies wurde bislang jedoch noch nicht untersucht.

Material und Methode: 141 PatientInnen mit primärer NNI und $150 \mathrm{~Pa}$ tientInnen mit sekundärer NNI, die an der Abteilung für Endokrinologie und Stoffwechsel, Medizinische Universität Wien, in Betreuung standen, wurden in eine Querschnittstudie eingeschlossen. Nach Abgleich mit dem Sterberegister wurden mittels Fragebögen die Häufigkeit einer Nebennierenkrise, die Notwendigkeit einer Dosisadaptierung der GC Therapie sowie potenzielle Auslöser erhoben. Eine Nebennierenkrise wurde als stationäre Aufnahme zur intravenösen GC Therapie definiert.

Ergebnisse: Von 141 PatientInnen ( $57 \pm 17$ Jahre) mit primärer NNI waren 9 verstorben, von 150 PatientInnen ( $60 \pm 16$ Jahre) mit sekundärer Nebenniereninsuffizienz waren 21 verstorben ( $6 \%$ vs $14 \% ; p=0,035$ ). Die verstorbenen PatientInnen waren signifikant älter $(72 \pm 12$ Jahre; $p<0,001)$. Bei 3 von 9 verstorbenen PatientInnen mit primärerer NNI und 1 von 21 mit sekundärer NNI konnte eine Nebennierenkrise als Todesursache nicht ausgeschlossen werden.

Die Fragebögen wurden von 86 PatientInnen mit primärer NNI (56 \pm 17 Jahre; $17 \pm 12$ Erkrankungsjahre) und 70 PatientInnen mit sekundärer NNI ( $56 \pm 16$ Jahre; $20 \pm 10$ Erkrankungsjahre) beantwortet. Nebennierenkrisen waren bei primärer NNI dreimal häufiger als bei sekundärer NNI ( $12 \pm 3$ vs $4 \pm 1$ Krisen pro 100 Patientenjahre; $p<0,001)$. Frauen hatten ein doppelt so hohes Risiko für eine Nebennierenkrise (Risk Ratio $2,05)$. Durchfall (29\%), Übelkeit (26\%), Fieber (25\%) und emotionaler Stress (25\%) waren die häufigsten Auslöser. In der jährlichen Adaptierungsrate der GC Dosis bestand kein Unterschied.

Schlussfolgerung: PatientInnen mit primärer NNI haben ein erhöhtes Risiko für Nebennierenkrisen. Die erhöhte Anzahl der verstorbenen PatientInnen mit sekundärer NNI ist vermutlich auf das höhere Alter und Komorbiditäten in dieser Untergruppe zurückzuführen. Frauen haben ein doppelt so häufiges Risiko für Nebennierenkrisen im Vergleich zu Männern.

\section{Erhöhtes Testosteron als unabhängiger Risikofaktor für Insulinresistenz bei Frauen mit und ohne PCOS}

Borzan V. ${ }^{* 1,2}$, Lerchbaum E. ${ }^{1}$, Missbrenner C. ${ }^{1}$, Heijboer A. C. ${ }^{3}$, Goschnik M. ${ }^{1,2}$, Trummer C.', Theiler-Schwetz V., Haudum C. ${ }^{1,2}$, Gumpold R. ', Schweighofer N. ${ }^{1,2}$, Obermayer-Pietsch B. ${ }^{1}$

${ }^{1}$ Medizinische Universität Graz, Graz, Österreich, ${ }^{2}$ Center for Medical Biomarker Research CBmed GmbH, Graz, Österreich, ${ }^{3}$ Vrije Universiteit, Amsterdam, Niederlande
Fragestellung: Das polyzystische Ovar-Syndrom (PCOS) ist mit einer Prävalenz von bis zu $22 \%$ aller Frauen weltweit eine der häufigsten Endokrinopathien im gebärfähigen Alter. Gemäß der derzeitigen klinischen Definition (zwei von drei „Rotterdam-Kriterien“) gibt es eine Vielzahl an Symptomen und Folgen, die im Zusammenhang mit erhöhten Androgenen stehen, darunter auch eine Insulinresistenz (IR). Eine größere Anzahl an Frauen weisen allerdings weniger als zwei Definitionskriterien auf - deren Risiko, Folgeerscheinungen wie IR oder Typ-2-Diabetes mellitus zu bekommen, wurde bisher kaum erforscht.

Material und Methoden: Wir führten eine retrospektive Analyse bei 750 Frauen mit und ohne PCOS durch. Dabei verglichen wir vier PCOS-Phänotypen mit Frauen, die nur ein Rotterdam-Kriterium aufwiesen und gesunde Kontrollen. Hormonelle und metabolische Unterschiede wurden mittels ANOVA beurteilt, zusätzlich führten wir logistische Regressionsanalysen durch.

Ergebnisse: Unter den PCOS-Phänotypen hatten Frauen mit Hyperandrogenämie ein erhöhtes Risiko für eine Insulinresistenz. Wir konnten insbesondere zeigen, dass Frauen mit nur einem Rotterdam-Kriterium ebenfalls ein erhöhtes Risiko für die Entwicklung von IR hatten, wenn dieses Kriterium eine Hyperandrogenämie war. Insbesondere bei erhöhtem freiem Testosteron zeigte sich ein bedeutend höheres Risiko für eine IR.

Schlussfolgerung: Unsere Ergebnisse legen nahe, dass beim PCOS eine Unterscheidung zwischen den Phänotypen zur Abschätzung des metabolischen Risikos entscheidend ist. Auch Frauen mit nur einem RotterdamKriterium sollten hinsichtlich einer Hyperandrogenämie, sowie einer potentiellen Insulinresistenz untersucht und gegebenenfalls therapeutisch beraten werden.

\section{Fehldiagnose Diabetes mellitus Typ 2}

Fegerl A. *', Leitgeb S. ', Hochfellner D. ', Mader J. K. ', Treiber G. 'Medizinische Universität Graz, Abteilung für Endokrinologie und Diabetologie, Graz, Österreich

Fragestellung: Neu auftretende Hyperglykämien im höheren Lebensalter werden aufgrund der Prävalenz meist dem Typ 2 Diabetes zugeschrieben. Das führt zu inadäquater diabetischen Stoffwechselkontrolle und kann auch schwerwiegende Folgen für den Patienten haben. Welche Relevanz die richtige Primärdiagnostik darstellt, wird anhand von drei Fällen präsentiert.

Material und Methode: Kasuistiken von 3 PatientInnen (A: Frau 48 Jahre (J), Diabeteslaufdauer (DL) 11, BMI: $21,9 \mathrm{~kg} / \mathrm{m}^{2}$ B: Frau 81 J, DL 1 J, BMI: 21,9 kg/m², C: Mann 51 J, Erstdiagnose, BMI 33,4 kg/m²).

Ergebnisse: Pat. A wurde unterlaufender 4-fach OAD (oraler antidiabetischer Therapie) inkl. SGLT-2 Hemmer mit einer schweren DKA (diabetischen Ketoazidose) aufgenommen. Die C-Peptidbestimmung ergab einen Wert von $0,17 \mathrm{ng} / \mathrm{ml}$, dies erforderte einen Regimewechsel auf eine funktionelle Insulintherapie.

Pat. B wurde nach Sekundärversagen der OAD Therapie inkl. SGLT-2 Hemmer im Rahmen einer OP-Vorbereitung vorgestellt. Weitere Abklärung zeigte ein C-Peptid von $0,92 \mathrm{ng} / \mathrm{ml}$ und GAD-AK von $>2000 \mathrm{U} / \mathrm{L}$, woraufhin eine funktionelle Insulintherapie eingeleitet wurde.

Pat. C mit vorbestehender Vitiligo, Zuweisung mit v. a. Typ 2 Diabetes bei $\mathrm{HbA} 1 \mathrm{c} 146 \mathrm{mmol} / \mathrm{mol}$. Weitere Abklärung zeigte ein C-Peptid (3,9 ng/ $\mathrm{ml}$ ), GAD-AK 1267 U/L und ZnT8-AK 820 U/L. Die initiale Insulintherapie plus Metformin wurde erweitert auf eine GPL-1 RA Therapie. Alle 3 Pat. wurden für T1D geschult und über das Risiko einer DKA ohne Insulintherapie bei abnehmender Betazellrestfunktion aufgeklärt.

Schlussfolgerung: Bei jedem Patienten mit BMI im Normalbereich oder vorbestehenden Autoimmunerkrankungen und neu aufgetretener Hyperglykämie empfiehlt es sich, eine weiterführende Diabetes Typisierung durchzuführen. Die frühzeitige Bestimmung von C-Peptid und der typischen T1D Autoantikörper helfen Fehldiagnosen und damit mögliche Risiken für die Patienten zu vermeiden. 
Hier steht eine Anzeige.

Springer 


\section{Ernährung und Hypoparathyreoidismus}

Fellrieser V. ${ }^{* 1}$, Tmava-Berisha A. ${ }^{2}$, Scherkl M. ${ }^{1}$, Fahrleitner-Pammer A. ${ }^{\text {, }}$, Amrein K. ${ }^{1}$

${ }^{1}$ Klinische Abteilung für Endokrinologie und Diabetologie, Universitätsklinik für Innere Medizin, Medizinische Universität Graz, Graz, Österreich, ${ }^{2}$ Universitätsklinik für Psychiatrie und Psychotherapeutische Medizin, Medizinische Universität Graz, Graz, Österreich

Fragestellung: Der chronische Hypoparathyreoidismus ist eine seltene Erkrankung, der mit einem verminderten Serumparathormonspiegel und einer daraus resultierenden Hypokalzämie, Hypercalciurie und Hyperphosphatämie einhergeht. Der Hypoparathyreoidismus wird konventionell mit Kalzium und Vitamin D behandelt, bei schwer einstellbaren PatientInnen wird auch rekombinantes Parathormon verwendet. Ungenügend geklärt ist, wie sich die Ernährung auf den Verlauf bei chronischen Hypoparathyreoidismus auswirkt.

Material und Methode: Mittels Literaturrecherche und Interviews mit Betroffenen wurde die Bedeutung der Ernährung im Management des chronischen Hypoparathyreoidismus evaluiert.

Ergebnisse: Bislang gibt es nur wenige publizierte Studien, aber insbesondere die Kalzium- als auch die Phosphatzufuhr in der Nahrung und in den Getränken ist im Alltag der Betroffenen klinisch relevant. Es ist sinnvoll und möglich, über ein Grundlagenwissen zum Thema Ernährung ein Empowerment zu erreichen.

PatientInnen mit einem Hypoparathyreoidismus sollten sich kalziumreich, phosphat- und natriumarm ernähren, zusätzlich sollte auf eine ausreichende Zufuhr von Magnesium und Vitamin D geachtet werden. Die Bioverfügbarkeit von Kalzium ist von sehr vielen Faktoren abhängig, wodurch manche Lebensmittel trotz des hohen Kalziumgehalts nicht dazu geeignet sind, um den Bedarf zu decken, auch eine gleichzeitige Einnahme mit Magnesium kann problematisch sein. Akute Symptome können statt mittels zusätzlichem Kalziumsupplement teils sehr gut durch Essen von einer kleinen Portion Käse (z. B. 20 g Parmesan) abgefangen werden. Auch das Timing von kalziumhältigen Lebensmitteln kann zu einer Verbesserung der Symptomatik führen (Fall 1 aus den Interviews).

Viele Fertigprodukte enthalten Phosphat in Form von sehr gut resorbierbaren Phosphatsalzen, was kurzfristig zu einer Entgleisung des KalziumPhosphat-Haushaltes führen kann (Fall 2 aus den Interviews).

Schlussfolgerung: Eine an die Grunderkrankung angepasste Ernährung spielt eine wichtige Rolle in der Kontrolle der Symptome und ist eine wichtige Basismaßnahme im Management des chronischen Hypoparathyreoidismus. Besondere Umstände wie bariatrisch Voroperierte oder von Malabsorption Betroffene erfordern teils eine Anpassung der Therapie.

\section{Evidenz für Blutglukosesenkung als Folge eines Erythropoietin- induzierten Hämatokritanstiegs in Mäusen}

Kaya A. ${ }^{1}$, Beghini M. ${ }^{1}$, Metz M. ${ }^{1}$, Kautzky-Willer A. ${ }^{1}$, Scherer T. ${ }^{1}$, Fürnsinn C. ${ }^{* 1}$ ${ }^{1}$ Klinik für Innere Medizin III, Abteilung für Endokrinologie \& Stoffwechsel, Medizinische Universität Wien, Wien, Österreich

Fragestellung: Wie im Vorjahr berichtet, senkt Erythropoietin (EPO) die Glykämie von adipösen Mäusen unabhängig von etwaigen Effekten auf das Körpergewicht. Die hier berichteten Untersuchungen dienten dem Verständnis des zugrundeliegenden Mechanismus der Blutglukosesenkung. Material und Methode: Männliche Mäuse, die durch fettreiche Diät adipös und hyperglykämisch waren, wurden mit drei intraperitonealen Injektionen pro Woche von 300 oder 1000 U/kg Erythropoietin behandelt. Dabei kamen Wild-Type-Mäuse (WT) zum Einsatz, sowie solche, bei denen der EPO-Rezeptor (EPOR) nur in Knochenmark und Milz exprimiert war, also nur in blutbildenden Organen (EPORko-TG). Um indirekte Effekte auf die Blutglukose durch Gewichtsverlust, wie er unter EPO-Exposition auftreten kann, zu vermeiden, wurden die Kontrollgruppen bei Bedarf durch eingeschränkte Fütterung auf dem Körpergewicht der jeweiligen EPO-behandelten Vergleichsgruppe gehalten.

Ergebnis: Zwei Monate Behandlung mit dreimal wöchentlich $300 \mathrm{U} / \mathrm{kg}$ EPO führte in WT-Mäusen zu einem Anstieg des Hämatokrits (Kontrolle,
$44,4 \pm 0,9$, vs EPO, $68,7 \pm 1,2 \%)$ und zu einer Reduktion der Nüchternblutglukose (Kontrolle, $168 \pm 8$, vs. EPO, $108 \pm 9 \mathrm{mg} / \mathrm{dl}, p<0,0001) .1000 \mathrm{U} /$ kg EPO (Hämatokrit 74,8 $\pm 0,5 \%$ ) hatte nicht nur anti-hyperglykämische Wirkung, sondern sogar einen deutlich hypoglykämischen Effekt, d.h. die Blutglukose sank weit unter den Normalwert von kohlenhydratreich gefütterten, schlanken Mäusen (Kontrolle adipös, $154 \pm 2$, vs. Kontrolle schlank, $110 \pm 4$, vs. EPO, $80 \pm 10 \mathrm{mg} / \mathrm{dl}$; jeweils $p<0,0002$ vs. EPO). Das Absinken der Blutglukose während der ersten zwei Behandlungsmonate verlief parallel dem Hämatokritanstieg langsam über die Zeit, was auf eine möglicherweise kausale Rolle der Blutbildung für das Absinken der Glykämie hinwies. Dementsprechend war die blutglukosesenkende Wirkung von EPO auch in EPORko-TG-Mäusen (Hämatokrit 63,3 $\pm 2,2 \%$ ) uneingeschränkt zu finden (Kontrolle, $168 \pm 4$, vs. $300 \mathrm{U} / \mathrm{kg}$ EPO, $91 \pm 9 \mathrm{mg} / \mathrm{dl}$, $p=0,0009)$.

Schlussfolgerung: Regelmäßige Behandlung mit EPO kann selbst bei adipösen, hyperglykämischen Mäusen die Nüchternblutglukose bis in den hypoglykämischen Bereich senken. Unsere Ergebnisse weisen darauf hin, dass diese blutglukosesenkende Wirkung von EPO durch Blutbildung und Hämatokritanstieg vermittelt werden könnte.

\section{Entwicklung der Schwangerschaftsverläufe mit präexistentem Diabetes Typ 1 und Typ 2 zwischen 2010-2019 an der Pregnancy Clinic eines tertiären Zentrums}

Häntschel E. ${ }^{* 1}$, Novak E. ${ }^{1}$, Stern C. ${ }^{2}$, Cervar-Zivkovic M. ${ }^{2}$, Vajda C. ${ }^{3,4}$, Treiber G. ${ }^{\prime}$ 'Medizinische Universität Graz, Universitätsklinik Innere Medizin, Abteilung Endokrinologie und Diabetologie, Graz, Österreich, ${ }^{2}$ Medizinische Universität Graz, Universitätsklinik für Frauenheilkunde und Geburtshilfe, Graz, Österreich, ${ }^{3}$ Medizinische Universität Graz, Universitätsklinik für Psychiatrie und Psychotherapeutische Medizin, Graz, Österreich, ${ }^{4}$ Univ.-Klinik für Medizinische Psychologie und Psychotherapie, Graz, Österreich

In der letzten Dekade ist eine Zunahme von Schwangerschaften mit Typ 1 Diabetes (T1D) und Typ 2 Diabetes (T2D) zu beobachten. Schwangerschaften bei Frauen mit T1D oder T2D haben ein erhöhtes Risiko für maternale und fetale Komplikationen. Ziel war es, Veränderungen in den maternalen Charakteristika sowie perinatalen Outcomes von Frauen mit T1D und T2D zwischen 2010 und 2019 zu erheben.

Methoden: 191 Schwangerschaften wurden untersucht und die Zeiträume 2010-2014 (58 T1D, 5 T2D) und 2015-2019 (83 T1D, 45 T2D) verglichen. Ergebnisse: HbAlc zu Schwangerschaftsbeginn in T1D (54 \pm 14 vs $51 \pm 13 \mathrm{mmol} / \mathrm{mol}$ ), Anteil mit HbAlc $<53$ (62\% vs $60 \%$ ), Hbalc 3 . Trimester ( $41 \pm 7$ vs $43 \pm 6 \mathrm{mmol} / \mathrm{mol}$ ) sowie der Anteil an diabetischen Folgeerkrankungen ( $21 \%$ vs $25 \%$ ) zeigten sich unverändert. Progression von diabetischer Retinopathie und/oder Nephropathie in der Schwangerschaft stieg von $3 \%$ auf $12 \%$. Weniger Frauen hatten eine präkonzeptionelle Beratung $(53 \%$ vs $29 \%, p<0.01)$. Der Einsatz von kontinuierlichen Glukosemesssystemen (FGM+CGM) nahm von $19 \%$ auf $77 \% \mathrm{zu}(p \leq 0.001)$, sowie der Anteil an Insulinpumpentherapie während der Schwangerschaft (36\% vs $46 \%, p<0.05)$. Die Sektiorate reduzierte sich von $60 \%$ auf $52 \%$, die Rate an LGA war ident bei $19 \%$ vs $19 \%$ und ähnlich bei Makrosomie (14\% vs $18 \%)$. Der Anteil an Frühgeburt vor der 37 . SSW lag bei $10 \%$ vs. $14 \%$ und Überwachung auf NICU lag bei $16 \%$ vs $19 \%$ ( $p=n s)$.

Frauen mit T2D hatten einen höheren BMI $\left(33,4 \pm 6,7 \mathrm{vs.} 25,1 \pm 6,7 \mathrm{~kg} / \mathrm{m}^{2}\right)$ und der Anteil an $\mathrm{HbAlc}<53 \mathrm{mmol} / \mathrm{mol}$ war mit $49 \%$ vs $60 \%$ geringer als bei T1D Frauen. Nur bei $38 \%$ der T2D Schwangerschaften kam es zu keinen maternalen oder fetalen Komplikationen.

Schlussfolgerungen: Es zeigte sich ein Anstieg von Schwangerschaften mit T1D und vor allem mit T2D. Der zunehmende Einsatz der Diabetestechnologie zeigte keine signifikanten Änderungen auf die diabetische Stoffwechselkontrolle. Ein Fokus ist auf eine vermehrte präkonzeptionelle Beratung bei T1D und T2D zu legen. 


\section{Kombinationstherapie mit Exenatide und Dapagliflozin zeigt keine synergistischen Effekte in der Leberfettreduktion trotz besserem Glukosemanagement bei Patienten mit T2 DM und Metformintherapie: EXENDA, eine prospektive, randomisiert- kontrollierte Studie}

Harreiter J. ${ }^{* 1}$, Just I. ${ }^{1}$, Leutner M. ${ }^{1}$, Brath H. ${ }^{2}$, Schelkshorn $C^{3}{ }^{3}$, Klepochova R. ${ }^{1}$, Krššák M. ${ }^{1}$, Kautzky-Willer A.

${ }^{1}$ Medizinische Universität Wien, Wien, Österreich, ${ }^{2}$ Gesundheitszentum Favoriten, Wien, Österreich, ${ }^{3}$ Landesklinikum Stockerau, Stockerau, Österreich

Fragestellung: Ziel dieser Studie war es, potentielle synergistische Effekte einer Kombinationstherapie aus Exenatide und Dapagliflozin (EXE+DAPA) vs. Placebo und Dapagliflozin (PLAC+DAPA) auf die Reduktion des hepatozellulären Fettgehalt (HF) nach 24 Behandllungswochen zu untersuchen.

Material und Methoden: Dreißig PatientInnen mit T2 DM wurden randomisiert und erhielten entweder EXE+DAPA $(n=16)$ oder PLAC+DAPA $(n=14)$. Einschlusskriterien waren HbA1c 6,5-11\%, Alter 18-75 Jahre $\mathrm{BMI} \geq 25 \mathrm{~kg} / \mathrm{m}^{2}$ und Metformin $\geq 1000 \mathrm{mg} /$ Tag. Der primäre Endpunkt HF wurde zu Beginn und nach 24 Wochen mittels Magnetresonanzspektroskopie untersucht. Zwischengruppeneffekte wurden mit allgemeinen linearen Modellen adjustiert für die Ausgangswerte, Alter, Geschlecht und BMI, Innergruppeneffekte mit gepaarten t-Tests untersucht.

Resultate: Nach 24 Wochen konnte eine Reduktion von HF in beiden Behandlungsgruppen beobachtet werden (absolut: EXE+DAPA -4,4\%, $95 \% \mathrm{CI}-8,2,-0,7, \mathrm{P}<0,05$; PLAC+DAPA -3,9\%, $95 \% \mathrm{CI}-6,0,-1,7$, $\mathrm{P}<0,01$; relativ: $\mathrm{EXE}+\mathrm{DAPA}-35,6 \%$, PLAC+DAPA -32,3\%). Zwischen den Gruppen gab es keine Unterschiede. Ähnliche Ergebnisse wurden für subkutanes (SAT) und viszerales Fettgewebe (VAT) beobachtet. HbA1c (EXE+DAPA: $-1,6 \%, 95 \% \mathrm{CI}-2,3,-1,0, \mathrm{P}<0,001$; PLAC+DAPA -0,6\%, $95 \% \mathrm{CI}-1,0,-0,3, \mathrm{P}=0,001)$ und Nüchternglukose waren signifikant niedriger in beiden Gruppen, obwohl in EXE+DAPA besseres Glukosemanagement erreicht werden konnte (HbAlc: $-0,55 \%, 95 \% \mathrm{CI}-0,88$, $-0,20, p<0,01)$. Das Körpergewicht war in beiden Gruppen geringer (EXE+DAPA -7,3 kg, $95 \% \mathrm{CI}-9,9,-4,8, \mathrm{P}<0,001$; PLAC+DAPA -4,6 kg $95 \%$ CI $-7,4,-1,8, \mathrm{P}<0,01)$, jedoch gab es zwischen den Gruppen keine Unterschiede. Positive Korrelationen fanden sich zwischen den Änderungen in HF und Gewicht, Hüft- und Bauchumfang, sowie VAT und SAT. Schlussfolgerung: Nach 24 Behandlungswochen war das Leberfett signifikant niedriger in EXE+DAPA und PLAC+DAPA, mit vergleichbaren Outcomes zwischen den Gruppen, trotz signifikant besserer Glukoseparameter in der EXE+DAPA Kombinationsgruppe. Änderungen im Leberfett waren assoziiert mit Gewichtsreduktion und Reduktion in VAT, aber nicht mit Glukoseparametern. Weitere Studien sind erforderlich, um potentielle synergistische Langzeiteffekte einer Kombinationstherapie zu untersuchen.

\section{Reversibility of neuropsychiatric symptoms and white matter changes after reinstitution of phenylalanine-free diet in an adult woman with PKU: a case report}

Beghini M. ${ }^{\prime}$, Milenkovic I. ${ }^{3}$, Christian L. ${ }^{2}$, Bergmann K. ${ }^{2}$, Scherer T. ${ }^{1}$, HufgardLeitner $M{ }^{* 1}$

'Division of Endocrinology, Department for Internal Medicine III, Medical University of Vienna, Austria, ${ }^{2}$ General Hospital of Vienna, Austria, ${ }^{3}$ Department for Neurology, Medical University of Vienna, Austria

Background: Phenylketonuria (PKU) is the most common congenital metabolic disorder (1:10.000 in Europe), and is included in the Austrian Newborn Screening Program since 1966. Since former recommendations supported the discontinuation of the phenylalanine-free diet at an age of 8-12 years, older PKU patients are often unaware that current guidelines recommend a life-long diet. Even when patients are symptomatic, they may not attribute their medical condition to PKU.

Case: In 2019, our outpatient clinic for Inborn Errors of Metabolism in adults (Medical University of Vienna) was contacted by a psychiatric clinic who referred a 39-year-old woman with severe depression, anxiety, and headaches. The patient had PKU and reported a discontinuation of her phe-free diet at the age of 14. Plasma phe-level was $1432 \mu \mathrm{mol} / \mathrm{l}$. In an initial Montreal Cognitive Assessment (MOCA) Test, the patient showed severe deficits in visuospatial function, delayed recall, abstraction and attention. A cMRI showed areas with marked T2 hyperintensity in the periventricular and parietooccipital white matter.

15 months after the reinstitution of a phe-free diet, phe- values declined to a mean value of $345 \mu \mathrm{mol} / \mathrm{l}$. The patient reported an improvement in her depressive symptoms, a decrease in the headache intensity and reduced intake of analgetics. The MOCA test showed significant improvement in visuospatial functions, delayed recall, abstraction and attention. After 15 months on a phe-free diet, the previously observed white matter T2 hyperintensities were undetectable.

Conclusions: This case report supports at least a partial reversibility of clinical manifestations due to high phe-levels in adult patients with PKU when an appropriate diet is reinstituted. Active efforts to reestablish contact with lost to follow up PKU adult patients unaware of current guidelines is therefore of highly clinical importance. Further research is needed to confirm whether white matter lesions are reversible when Phe-levels are lowered to "near normal" levels.

\section{Individualisierte, physiologische,pulsatile-GnRH-Therapie bei idiopathischem Hypogonadotropem Hypogonadismus - Ein Durchbruch?}

Kunz $Y^{*}{ }^{* 1}$, Wurzacher J. ${ }^{1}$, Pinggera $G .^{1}$

'Univ. Klinik für Urologie, LKH Innsbruck, Innsbruck, Österreich

Fragestellung: Der idiopathische hypogonadotrope Hypogonadismus (IHH) ist mit einer Inzidenz von 1: 10.000-1:100.000 eine relevante Ursache männlicher Infertilität, gekennzeichnet durch eine gestörte Sekretion des Gonadotropin-Releasing-Hormons ( $\mathrm{GnRH})$ im Bereich des Thalamus. Die dadurch verursachte ineffiziente Ausschüttung von Luteinisierendem Hormon (LH) und Follikelstimulierendem Hormon (FSH) in der Hypophyse führt zu erniedrigten Testosteronwerten und sekundärer Infertilität. Unterschieden wird die X-chromosomal rezessive Variante beim Kallman-Syndrom, mit Anosmie, von weiteren, genetisch bedingten IHH Varianten. Pulsatilität in der GnRH-Sekretion oder die Sekretionsamplitude ist entscheidend für eine adäquate testikuläre Antwort auf endokrinologisch-reproduktiver Ebene.

Material und Methode: Bei fünf Patienten (mittleres Alter: 26) mit unerfülltem Kinderwunsch wurde die Diagnose IHH gestellt (mean T 0,12 ng/ $\mathrm{ml}$, LH 0,32 U/l, FSH 0,68 U/l). Alle Patienten wiesen eine Azoospermie, drei Patienten zusätzlich eine Anejakulation, auf. In der Therapie wurden sie in den Gebrauch der GnRH-Pumpe (LutrePulse ${ }^{\circledast}$ ) geschult. Eine pulsatile $\mathrm{GnRH}$-Therapie wurde mit 10 bis $20 \mu \mathrm{g}$ alle 120 Minuten initiiert. Verlaufskontrollen mit Spermiogramm und Hormonstatus wurden nach vier und zwölf Wochen, und in zwei Fällen nach 6 Monaten durchgeführt. Die Medikations-Compliance wurde vierwöchentlich überprüft.

Ergebnisse: Nach vier Wochen zeigten alle Patienten einen signifikanten Anstieg der Testosteronwerte und der Gonadotropine (mean T 3,35 ng/ $\mathrm{ml}$, LH 4,46 U/l, FSH 5,7 U/1). Nach drei Monaten konnten bei allen Patienten eugonadale Werte festgestellt werden (mean T 5,42 ng/ml, LH 3,52 U/l, FSH 5,9 U/l). Bei drei Patienten mit vorheriger Anejakulation zeigte sich sogar keine Ejakulationsdysfunktion mehr. 3/5 der Patienten erzielten eine Oligoasthenoteratozoospermie (OAT), sowie eine Normozoospermie, resultierend in erfolgreicher Kryokonservierung für ART, einmal mittels Mikro-TESE. Interessanterweise konnte bei drei Patienten eine bis zu vierfache Zunahme des Hodenvolumens festgestellt werden.

Schlussfolgerung: Die pulsatile GnRH Verabreichung optimiert erfolgreich in physiologischer Weise die IHH Therapie. Nach sechs Monaten konnte bei allen fünf Patienten eine Kryokonservierung abgeschlossen werden. Unsere Serie zeigt den einfachen und verlässlichen Einsatz dieser Pumpe im klinischen Alltag. 


\section{A new analytical method for the determination of the Paraxanthine/Caffeine ratio as a metabolic biomarker for CYP1A2 activity by UHPLC-ESIMS/MS}

Lajin B. ${ }^{* 1}$, Schweighofer N. ${ }^{1}$, Goessler W. ${ }^{2}$, Obermayer-Pietsch B. ${ }^{1}$

'Division of Endocrinology and Diabetology, Department of Internal Medicine, Medical University of Graz, Graz, Austria, ${ }^{2}$ Institute of Chemistry, Analytical Chemistry for the Health and Environment, University of Graz, Graz, Austria

Introduction: The Cytochrome P450 CYP1A2 is a central enzyme in the metabolism of drugs and xenobiotics in humans. The overall activity of this enzyme is influenced by a complex array of biochemical, dietary, and genetic factors. One of the simplest ways to probe the overall output of CYP1A2 is to measure the ratio between the concentration of a precursor and a product of its activity. The Paraxanthine/Caffeine ratio following controlled intake of caffeine serves as an attractive candidate and has been proposed and utilized for this function.

Aim: With the growing interest in the Paraxanthine/Caffeine ratio, the need arises to develop improved analytical methods specifically for the rapid and sensitive determination of paraxanthine and caffeine in biological samples. We report a new method optimized for the simultaneous determination of caffeine and paraxanthine in various human matrices. Materials and methods: The method involved sample pretreatment with ethanol precipitation and was based on ultra-high performance liquid chromatography separation coupled with tandem mass spectrometric detection (UHPLC-ESIMS/MS).

Results: The developed method offers a low limit of detection $(0.1 \mathrm{pg})$, rapid chromatographic separation (ca. $5 \mathrm{~min}$ ), the utilization of a green chromatographic solvent ( $5 \%$ ethanol), direct determination with minor sample preparation, and the employment of isotopically labeled internal standards and qualifier ions to ensure best accuracy. Validation in urine, saliva, and plasma was performed by spiking experiments where the recovery and repeatability were within $\pm 15 \%$ and $\pm 10 \%$, respectively. The method was applied in a group of volunteers following controlled caffeine administration.

Conclusion: The developed method is optimized for the simultaneous determination of paraxanthine and caffeine, offers several advantages over the current methods (including 10-fold improvement in the limit of detection), and is suitable for application in large clinical studies.

\section{Testosterone deficiency is a risk factor for severe SARS-CoV-2 infections in men}

Lanser L. ${ }^{* 1}$, Burkert F. R. ${ }^{1}$, Thommes L. ${ }^{1}$, Egger A. ${ }^{2}$, Hoermann G. ${ }^{2}$, Anliker M. ${ }^{2}$ Griesmacher A. ${ }^{2}$, Kaser S. ${ }^{3}$, Pinggera G.-M. ${ }^{4}$, Weiss G. ${ }^{1}$, Bellmann-Weiler R. ${ }^{1}$ Medical University of Innsbruck, Department of Internal Medicine II, Innsbruck, Austria, ${ }^{2}$ Innsbruck University Hospital, Central Institute for Medical and Chemical Laboratory Diagnosis, Innsbruck, Austria, ${ }^{3}$ Medical University of Innsbruck, Department of Internal Medicine I, Innsbruck, Austria, ${ }^{4}$ Medical University of Innsbruck, Department of Urology, Innsbruck, Austria

Introduction: Men were shown to have more severe clinical manifestations of SARS-CoV-2 infections although the absolute numbers of confirmed cases are similarly in men and women. Beside increased prevalence of comorbidities, sex hormones were suggested to affect disease progression men with COVID-19.

Material \& Methods: We studied for effects of sex hormones on disease severity and their relations to immune activation in 155 men with PCRconfirmed SARS-CoV-2 infections who were hospitalized at the Innsbruck University Hospital between February and December 2020. Clinical outcomes during hospitalization as well as demographics, clinical and laboratory parameters were collected.

Results: Men presented with rather low baseline serum total testosterone (tT) levels upon hospital admission while luteinizing hormone (LH) and estradiol (E2) were within the normal range. Lower baseline tT levels were associated with a more activated immune system, as reflected by elevated levels of the biomarkers C-reactive protein (CRP), interleukin 6 (IL-6) or procalcitonin levels, lower cholesterol levels and an increased risk for death or ICU admission during hospital stay. Men with tT levels $<100 \mathrm{ng} /$ $\mathrm{dL}$ (lowest tertile) had a more than nineteen-fold higher risk to die during hospital stay when compared to men with $\mathrm{tT}$ levels $>220 \mathrm{ng} / \mathrm{dL}$ (highest tertile). Interestingly, also higher E2 levels and a lower tT/E2 ratio upon hospital admission were associated with a higher risk for death or ICU admission during hospital stay.

Conclusion: Men hospitalized due to COVID-19 present with rather low testosterone levels accompanied by increased immune activation and more severe disease course with an increased in-hospital morbidity and mortality. Testosterone deficiency might primarily originate from altered cholesterol biosynthesis due to SARS-CoV-2 infection and deteriorate following inflammation-induced gonadotrophin suppression.

\section{Maternal and pregnancy outcome in women with gestational Diabetes Mellitus}

Merza Y. ${ }^{* 1}$, Harreiter J. ${ }^{1}$, Kautzky-Willer A. ${ }^{~}$, Itariu B.-K. ${ }^{1}$ ${ }^{1} A K H$ Wien, III. Medizinische Abteilung, Wien, Österreich

Up to $90 \%$ of pregnancies complicated by diabetes are affected by Gestational Diabetes Mellitus (GDM). The importance of aiming to understand and effectively treat or prevent GDM is illustrated by the wide-ranging maternal and fetal consequences of GDM.

The aim of this study was to retrospectively investigate outcomes of pregnancies in women aged 18 to 50 years who were diagnosed with GDM and under medical care at the diabetes outpatient department of the Department of Endocrinology and Metabolisms at the Medical University of Vienna and the Vienna General Hospital between 2014 and 2017, depending on their BMI and HbAlc. Several fetal and maternal parameters were included. In order to identify differences regarding the pregnancy outcome and complications maternal parameters of 214 pregnant women were compared and correlations were investigated.

The majority (62\%) of the patients had a BMI $\geq 30 \mathrm{~kg} / \mathrm{m}^{2}$. Obesity in pregnant women diagnosed with GDM was not only associated with an excessive weight gain during pregnancy, but also with an adverse outcome such as Cesarean section $(45.7 \%$ vs. $61.7 \%, p=0.023)$ and macrosomia $(10.1 \%$ vs. $23.9 \%, p=0.007)$. Furthermore, the majority (73\%) of the study population was treated with insulin, representing a higher than average insulinisation rate. Additionally, expectant mothers with an adverse outcome (LGA, admission to NICU, perinatal death, malformation, preeclampsia, premature rupture of membranes or vacuum extraction) had a statistically significant higher HbA1c at admission $(5.29 \pm 0.41$ vs. $5.46 \pm 0.72, p=0.015)$ and before pregnancy $(5.36 \pm 0.39$ vs. $5.57 \pm 0.64, p=0.011)$.

To prevent these adverse outcomes an early metabolic control and lifestyle interventions, ideally even before pregnancy, are of utmost importance. Further research is necessary to evaluate the usefulness of $\mathrm{HbAlc}$ as a predictor for adverse outcome in GDM.

\section{Oral sensory stimulation increases hepatic VLDL triglyceride secretion in humans}

Metz M. ${ }^{* 1}$, Wolf P. ', Beghini M. ', Bastian M. ${ }^{1}$, Hackl M. ${ }^{1}$, Baumgartner-Parzer S. ${ }^{1}$, Kautzky-Willer A. ${ }^{1}$, Trauner M. ${ }^{2}$, Marculescu R. ${ }^{3}$, Krebs M. ${ }^{1}$, Krššák M. ${ }^{4}$, Stangl $H^{5}{ }^{5}$, Fürnsinn $C^{1}{ }^{1}$, Scherer $T .{ }^{1}$

'Division of Endocrinology and Metabolism, Department of Internal Medicine III, Medical University of Vienna, Vienna, Austria, ${ }^{2}$ Division of Gastroenterology and Hepatology, Department of Internal Medicine III, Medical University of Vienna, Vienna, Austria, ${ }^{3}$ Department of Laboratory Medicine, Medical University of Vienna, Vienna, Austria, ${ }^{4}$ Centre of ExcellenceHigh Field MR, Department of Biomedical Imaging and Image-guided Therapy, Medical University of Vienna, Vienna, Austria, ${ }^{5}$ Institute of Medical Chemistry, Center for Pathobiochemistry and Genetics, Medical University of Vienna, Vienna, Austria

The autonomic nervous system, which connects the brain with the liver, regulates hepatic lipid and glucose metabolism in rodents. However, its 
role in human lipid metabolism remains to be elucidated. In this randomized, placebo-controlled crossover trial, we investigated the effects of modified sham feeding (MSF) on hepatic very low-density lipoprotein (VLDL) triglyceride secretion in 10 healthy, overnight-fasted, male subjects (age $26 \pm 3$ yrs; BMI $24.2 \pm 2.5$ ). MSF is an established method to rapidly induce cephalic phase vagus nerve activity, where subjects smell, taste and chew, but do not swallow a test meal. We assessed VLDL triglyceride secretion using an intralipid infusion test combined with density gradient ultracentrifugation to isolate accumulating large liver-derived VLDL1 particles over time. Cephalic phase pancreatic polypeptide (PP) secretion served as a vagal activation marker. We show that MSF robustly increases VLDL triglyceride secretion $(244 \pm 39 \mathrm{mg} / \mathrm{h}$ vs. $348 \pm 32 \mathrm{mg} / \mathrm{h} ; p=0.02)$ and plasma PP levels (4-fold increase; $p=0.02$ ) compared to control conditions, where water was chewed instead. Notably, plasma insulin and glucose levels were unchanged. These results suggest that the vagus nerve contributes to the regulation of hepatic lipid metabolism in humans as well. We demonstrate a novel cephalic phase reflex that likely prepares the liver for newly incoming lipids by increasing hepatic VLDL export a key mechanism that protects the liver from ectopic lipid accumulation and steatosis.

\section{A Phase 3 Study of a Modified-Release Hydrocortisone in the Treatment of Congenital Adrenal Hyperplasia}

Bendfeldt H. ${ }^{1}$, Mallappa A. ', Arlt W. ${ }^{2}$, Brac de La Pierre A. ${ }^{3}$, Linden Hirschberg A. ${ }^{4}$, Juul A. ${ }^{5}$, Newell-Price J.D. C. ${ }^{6}$, Perry C. G. ${ }^{7}$, Prete A. ${ }^{2}$, Rees A. ${ }^{8}$, Reisch N. ${ }^{* 9}$, Stikkelbroeck M. ${ }^{10}$, Touraine P. A. ${ }^{11}$, Maltby K. ${ }^{12}$, Treasure P. ${ }^{13}$, Porter J. ${ }^{12}$, Ross R.J.M. ${ }^{6}$

${ }^{1} \mathrm{NIH}$, Bethesda, USA, ${ }^{2}$ University of Birmingham, Birmingham, United Kingdom, ${ }^{3}$ Hopital Louis Pradel, Bron, France, ${ }^{4}$ Karolinska Institutet, Stockholm, Sweden, ${ }^{5}$ RIGSHOSPITALET, Copenhagen, Denmark, ${ }^{6}$ University of Sheffield, Sheffield, United Kingdom, ${ }^{7}$ Queen Elizabeth University Hospital, Glasgow, United Kingdom, ${ }^{8}$ Cardiff University, Cardiff, United Kingdom, ${ }^{9}$ Medizinische Klinik und Poliklinik IV, Klinikum der Universität München, München, Germany, ${ }^{10}$ Radboud Univ Nijmegen Med Ctr, Nijmegen, The Netherlands, ${ }^{11} \mathrm{GH}$ Pitie Salpetriere, Paris Cedex 13, France, ${ }^{12}$ Diurnal Ltd., Cardiff, United Kingdom, ${ }^{13}$ Statistical Services Ltd, Kings Lynn, United Kingdom

Background: Patients with congenital adrenal hyperplasia (CAH) due to classic 21-hydroxylase deficiency have poor health outcomes related to inadequate glucocorticoid (GC) replacement. We compared disease control of adults with classic $\mathrm{CAH}$ treated with a modified release hydrocortisone (MRHC) replicating physiological cortisol secretion, versus standard GC therapy.

Methods: 6 month, open label, study in 122 patients randomised either to treatment with MRHC (Chronocort ${ }^{\circledR}$, Diurnal Ltd, Cardiff, UK) twice daily at $\sim 0700 \mathrm{~h} \& \sim 2300 \mathrm{~h}$, or to remain on their standard GC regimen. Patients had 24-hr profiling of serum 17-hydroxyprogesterone (17-OHP) at baseline and for dose titration at 4 and 12 weeks. The primary efficacy endpoint was the change from baseline to 24 weeks in the natural logarithm of the mean of the 24-hr standard deviation score (SDS) profile for 17-OHP Results: Both groups improved hormonal control at 24 weeks. The mean 24-hour 17-OHP SDS was significantly lower on MRHC compared to standard GC at 4 weeks $(p=0.0074)$ and 12 weeks $(p=0.019)$, but not at 24 weeks. At 24 weeks, MRHC treatment showed greater reduction in 17-OHP SDS compared to standard GC in the morning, 0700-1500 h $(p=0.0442)$ and a greater reduction in log transformed 17-OHP $24 \mathrm{~h}$ AUC $(p=0.0251)$. The variability of $17-\mathrm{OHP}$ over 24 hours was significantly reduced in the MRHC group compared to standard GC. There were no adrenal crises on MRHC (3 in the standard GC group) and fewer stress doses. Patient reported benefit included restoration of menstruation in 4 patients on MRHC and 1 on standard GC and two partner pregnancies in patients on MRHC and none on standard GC.

Discussion: Intensification of therapy improved control of 17-OHP. MRHC reduced the fluctuations in 17-OHP providing consistent, optimal disease control.
Conclusion: MRHC improves the biochemical control of classic CAH with a twice-daily therapy.

\section{miRNA Signaturen bei Immunthyreopathie Hashimoto}

Trummer . $^{* 1}$, Arifi E. ', Schweighofer N. ${ }^{1,2}$, Trummer C. ', FößII.', Haudum C. W. ${ }^{2}$, Obermayer-Pietsch B. ${ }^{1}$

${ }^{1}$ Medizinische Universität Graz, Graz, Österreich, ${ }^{2} \mathrm{CBmed} \mathrm{GmbH}$, Graz, Österreich

Hintergrund: Die Immunthyreopathie Typ Hashimoto zählt zu den häufigsten Autoimmunerkrankungen. Eine Immunreaktion führt zur Infiltration von T-Lymphozyten ins Parenchym der Schilddrüse und setzt, begleitet von spezifischen Autoantikörpern gegen Schilddrüsen-Proteine, eine chronische Entzündung in Gang.

MicroRNAs (miRNAs) sind kurze, einzelsträngige, nicht codierende RNASequenzen, die die Genexpression auf sekundärer Ebene regulieren, indem sie an mRNA binden und damit in die Translation eingreifen. Bei immunologischen Prozessen wie der Leukozyten-Aktivierung, der Proliferation, der Apoptose oder Zytokin-Expression können sie eine entscheidende Rolle spielen und wurden für die Immunthyreopathie Hashimoto von Martínez-Hernández et al. 2018 hinsichtlich ihrer Assoziationen untersucht.

Ziel unserer Untersuchung ist der Vergleich von miRNA-Profilen von Personen mit Immunthyreopathie zu gesunden Kontrollen aus der BioPersMed-Kohorte (Biomarker for Personalised Medicine).

Material und Methoden: Eine RNA-Isolierung und qPCR-Messung der Expression für 9 miRNAs erfolgte bei ausgewählten Patient*innen mit Immunthyreopathie Hashimoto $(n=26)$, sowie bei 18 gesunden Kontrollen aus der BioPersMed-Kohorte. Die miRNAs wurden mittels miRCURY miRNA PCR Assays (Qiagen) detektiert. Die statistische Auswertung erfolgte in IBM SPSS 25 .

Ergebnisse: Im Serum von Hashimoto Patient ${ }^{*}$ innen fanden wir miRNA$21-5 p(p=0,031)$, sowie miRNA-375 $(p=0,003)$ niedriger exprimiert als in gesunden Kontrollen, wohingegen miRNA-22-5p $(0,035)$ und miRNA-451 $(<0,001)$ im Vergleich höhere Expressionen zeigten.

Schlussfolgerungen: MiRNA-21-5p, miRNA-375 und miRNA-451 stellen interessante neue potentiell prädiktive Biomarker für Diagnostik und Monitoring einer Immunthyreopathie dar und werden nun in großen Kohorten und im Verlauf der Erkrankung untersucht.

\section{A case of adrenocortical carcinoma without endocrine alterations}

Zitterl A. ${ }^{* 1,2}$, Peric S. ${ }^{1,2}$, Prager M. ${ }^{3}$, Kreuzwieser E. ${ }^{1}$, Langer J. ${ }^{4}$, Stulnig T., ${ }^{1,2}$

13. Medizinische Abteilung mit Stoffwechselerkrankungen und Nephrologie, Klinik Hietzing, Wien, Österreich, ${ }^{2}$ Karl-Landsteiner-Institut für Stoffwechselerkrankungen und Nephrologie, Wien, Österreich, ${ }^{3} 2$. Chirurgische Abteilung, Klinik Hietzing, Wien, Österreich, ${ }^{4}$ Jakob-Erdheim-Institut für Pathologie und klinische Bakteriologie, Klinik Hietzing, Wien, Österreich

Introduction: We report a case of large adrenocortical carcinoma in an oligosymptomatic patient that developed within less than a year.

Case course: A fifty-one-year-old woman presented to a radiologic office for a CT scan due to a history of right-sided flank pain. A lesion with $6.5 \mathrm{~cm}$ in diameter in the right adrenal gland was found, denting liver and kidney. The lesion was not visible in a sonographic examination eight months before.

The patient was referred to an endocrinology department for further workup. The patient had a history of drug-induced headache and hypertension (well controlled with 2 antihypertensive agents). Hormonal testing was unremarkable with suppressible glucocorticoid axis and (nor-)metanephrines within normal range. No clinical signs of androgen excess were observed. Due to tumor size the patient was referred to the surgical ward for adrenalectomy. Intraoperatively, the mass could not be separated from the kidney, which necessitated en-bloc resection. No pathologically enlarged lymph nodes were noted. Histological workup found an adrenocortical carcinoma (ACC) measuring $8 \times 8 \times 5 \mathrm{~cm}$ and fulfilling $8 / 9$ Weiss scoring criteria for adrenal malignancies (UICC classification: pT2 L0 V1 
R0) but no infiltration of the kidney. Ki67 index was approximately $40 \%$, denoting a high risk for recurrence. Neither locoregionary nor distant metastases were found. Postoperatively the patient developed acute kidney injury but recovered completely. Adjuvant mitotane therapy for two years and regular follow-ups were decided for further management.

Discussion: ACCs are exceptionally rare tumors whose management requires endocrinological, surgical and histopathological expertise. Contrary to this case, most ACCs become symptomatic by excessive hormone production which must be tested prior to therapy. Differentiation between malignant and benign tumors is difficult and based on Weiss score whose assessment may be compromised by unsuccessful surgery. Despite improvements in medical management ACC still carries a poor prognosis with a median overall survival of 3-4 years.

Hinweis des Verlags. Der Verlag bleibt in Hinblick auf geografische Zuordnungen und Gebietsbezeichnungen in veröffentlichten Karten und Institutsadressen neutral. 